\title{
Drogadicción y Prevención familiar: una política para Europa
}

\author{
Fernando J. Mendes \\ Presidente IREFREA Portugal
}

Enviar correspondencia a:

Fernando Mendes. Av. Joao de Deus Ramos, 130-A,1 dto. 3030 Coimbra (Portugal) E-mail: irefrea@esoterica.pt

\section{Resumen:}

Se discute la necesidad de adoptar lo más rápidamente posible una política europea de prevención primaria de las drogadicciones a nivel familiar. El concepto de familia, su organización y estructura son hoy puestos en causa, tal y como los modelos y prácticas educativas. Se apunta hacia un mayor conocimiento y mayor investigación en este campo de la familia que permitirá el delineamiento de programas de prevención más precisos y eficientes en el futuro.

Se aborda igualmente la discusión en torno al concepto de prevención primaria y de los modelos teóricos que son la base de las prácticas preventivas, teniendo en cuenta los objetivos a alcanzar y las estrategias a utilizar.

Finalmente, a partir del resultado de una investigación del IREFREA en 4 países europeos (Portugal, España, Francia, Italia) se propone la elaboración de un Vademecum europeo sobre prácticas preventivas en Europa.

Palabras clave: prevención primaria, prevención familiar; familia; factores de protección; factores de riesgo, políticas preventivas.

\section{Summary:}

It is being discussed the need to establish, as quick as possible, an European addiction primary prevention policy, at family level.

The family concept, it's organisation and structure are nowa-days, questioned, abreast with the education practices. The aim is the highest knowledge and investigation, in this familiar area, which will allow to draw, in the future, more precise and efficient prevention programs.

It's broached, also the discussion about the primary prevention concept and the theoretical models which support the preventing practices, having in mind the final objectives and the used strategies.

This article does resume some of the risk factors and protection factors existent in the literature.

Finally, starting from the result of one IREFREA's investigation made in four countries (Portugal, Spain, France and Italy), it's proposed the elaboration of an european Vademecum about the european preventive practices.

Key words: primary prevention, family prevention, protective factors, risk factors, prevention policies.

\section{INTRODUCCIÓN}

E n el umbral del Siglo XXI el problema del consumo de sustancias no parece querer confinarse al pasado reciente y entramos en el nuevo siglo con uno de los más serios problemas de salud pública. Y esto ocurre a pesar de toda la investigación llevada a cabo y de los trabajos realizados en los últimos 30 años, en el campo de la prevención del uso/abuso de sustancias lícitas (alcohol y tabaco) e ilícitas.

Conocer las razones y factores que han originado directa o indirectamente el problema constituye la preocupación de todos los que quieren hacer de la prevención una verdadera ciencia. Enfrentándonos a resultados unas veces poco concluyentes, otras, insuficientemente satisfactorios, de las diversas estrategias utilizadas en esta área, hemos evolucionado paulatinamente hacia una mayor participación de las familias.

Sin embargo, estas tentativas de hacer que las familias participen están todavía lejos de ser una realidad que se traduzca en programas, proyectos o intervenciones, en número y calidad suficiente para que puedan ser reconocidos, divulgados y utilizados, al menos en los países de la Comunidad Europea. Si la familia es reconocida como la estructura en cuyo seno enseñamos y transmitimos a los niños y adolescentes nuestras creencias, ideas y valores, será con ella con la que tendremos que trabajar, con el objetivo de desarrollar instrumentos que les permitan a los padres "ajustar" y desarrollar algunos de sus comportamientos y actitudes ya en las primeras 
etapas del desarrollo del ciclo vital de la familia, dotando a los más jóvenes de mecanismos de defensa que hagan frente a la presión creciente del consumo de sustancias.

La mejor comprensión del proceso de desarrollo humano en su globalidad nos enseña que el proceso de socialización del niño es uno de los primeros y principales factores que predicen el comportamiento infantil, reforzando así el interés en intervenir muy pronto en la familia. (Macoby, 1992; Kumpfer y al., 1989).

Pero también al hablar del concepto "familia" en este final de siglo tenemos que considerar que, tanto la estructura familiar como la realización de los papeles parentales, ya no son los que conocíamos. Todas las transformaciones económicas, sociales y laborales que han tenido lugar a partir de los años 40 , junto con el cambio del papel de la mujer, con una participación cada vez más activa en la sociedad, han contribuido a alterar este concepto. También se cuestionan hoy en día los modelos y prácticas educativas que parecen responder mal a la presión más intensa para el consumo de las nuevas y viejas drogas.

Así, la acción y programación futuras en este campo tienen que tener en cuenta estas nuevas realidades si quieren responder y contribuir a una intervención más real y eficiente.

\section{CONCEPTOS DE PREVENCIÓN PRIMARIA}

El objetivo de la prevención primaria es básicamente el de evitar,. disminuir o reducir el impacto y las consecuencias negativas de determinadas situaciones y comportamientos, tanto a nivel individual como social.

Coie y col. (1993), redefiniendo este concepto, lo presentan como una nueva ciencia en un punto de unión de la psicología, la criminología, la, psiquiatría y el desarrollo humano. La prevención se presenta así como la solución al problema de la drogadicción - intervenir/actuar - incluso antes de la existencia del problema. Esta perspectiva presupone una nueva alternativa de observar, comprender e intervenir para que las situaciones no surjan, no se desarrollen, o no se repitan.

En esta línea de trabajo algunos autores abordan esta perspectiva a partir de la comprensión y conocimiento de los factores que actúan precozmente y que pueden llevar a la adición (Bergeret, J., 1982); para otros la prevención es un proceso activo que intenta adoptar y desarrollar iniciativas, y que tiene como objetivo modificar y perfeccionar la formación integral y la calidad de vida del individuo, aumentando su autodominio y su resistencia a la tentación de las drogas. (Martin, 1993).

Pero entre los investigadores y otros profesionales que trabajan en este campo, el concepto de prevención primaria de la drogadicción no ha sido consensuado, lo que ha provocado cierta perturbación en la elaboración de los programas, de la definición de los objetivos a alcanzar, de las metodologías utilizadas y de su aplicabilidad. Surgen por ejemplo cuestiones del tipo sobre quién actuar (grupo que constituye el blanco directo o indirecto) y qué tipo de acciones o programas pueden y deben ser utilizados.

En 1987 Gordon proponía tres formas distintas de intervención (adoptadas en 1994 por el American Institute of Medicin), teniendo en cuenta la valoración del riesgo y los componentes de los programas: prevencion universal, prevención selectiva y prevención específica.

Los programas de prevención universal se dirigen a la población en general, incluyendo a jóvenes y familias. Sus objetivos son informar y sensibilizar en relación a la problemática del consumo de sustancias. Son ejemplos las campañas de prevención de los media, los programas de intervención escolar y algunos programas de consolidación de las familias (Preparing for the drug. Free years Program, Hawkins y al., 1996; lowa Strenghtening Families Program, Molgaard y Kumpfer, 1995, FAST, McDonald, 1996, entre otros).

Los programas de prevención selectiva se dirigen a grupos o familias en riesgo "estadístico", dada la mayor probabilidad de llegar a consumir o de generar consumos. Estas intervenciones afectan a toda la familia, durante largos periodos (Strenhtening Families Program, Kumpfer y al., 1989)

Los programas de prevención específica se dirigen a grupos de riesgo bien definidos: familias en que se identifican o diagnostican, además de los factores de riesgo no específicos, otros como: fracaso escolar, disfunción familiar, comportamientos de delincuencia, consumo de drogas, abuso físico o sexual.

Para muchos autores la prevención primaria de las drogadicciones puede insertarse en una perspectiva de "Promoción de la salud" y de "Educación para la salud", con programas globales, muchas veces vagos, impreci- 
sos y demasiado vastos en sus objetivos y contenidos, integrando y diluyendo la especificidad o singularidad del tema de las drogas. Otros como Calafat, A. (1995), defienden la especificidad de las intervenciones en la prevención - "La prevención específica será toda la acción, actividad o programa que aborde el tema de una manera clara, concreta y explícita". Esta cuestión de la especificidad de una intervención preventiva ha sido mal comprendida por muchos técnicos en este campo ya que la consideran más una acción promotora de consumos, dado su fuerte componente informativo, reduccionista en relación a la información sobre las sustancias y sus efectos. Sin embargo, la prevención específica combina la información sobre los efectos de las sustancias con técnicas de control y protección contra la presión exterior, desarrollo del espíritu crítico y clarificación de valores (dinámica de grupos y entrenamiento de situaciones)

Los resultados de los estudios e investigación en curso, por lo que respecta a la aplicabilidad de los programas de prevención específica, nos permitirán a corto plazo una valoración más fundamentada. En los últimos años las intervenciones preventivas han usado una variedad de acercamientos y técnicas, desde "técnicas alarmistas" a modelos educativos de refuerzo de la autoestima, toma de decisiones, desarrollo de modelos personales y sociales, desarrollo de sentido crítico, habilidad para el aumento de la capacidad de comunicación interna (reconocimiento de nuestras emociones y sentimientos) y externa (verbalización y comprensión de los demás), afirmación y refuerzo del poder de resistencia a la presión de los grupos de iguales, entre otros.

Evidentemente, la riqueza y diversidad culturales, la diferencia de modelos educacionales entre la Europa Mediterránea y la Europa del Norte, así como otros muchos factores, tendrán que ser considerados en los futuros programas a "delinear", ya que prevenir es en esencia: informar - sensibilizar - ensayar - educar - modificar ofrecer alternativas - promover estilos de vida saludables.

\section{LA PREVENCIÓN FAMILIAR}

Una simple frase publicitaria cargada de sentido e intención, “La tradición ya no es lo que era”, ilustra bien el sentimiento y la perspectiva que tenemos sobre lo que consideramos que son "verdades absolutas e incuestionables". El aprovechamiento de esta frase, hecho en particular por los más jóvenes, ha servido de justificación para comportamientos y actitudes que rompen con lo que hacíamos, conocíamos o pensábamos que era mejor, para nosotros y para los demás. Ahora se cuestionan los modelos educativos, se ponen en causa los valores actuales, se discuten prácticas educativas y se valora o se devalúa la calidad y cantidad relacional en el seno de la familia. Se instala lentamente una actitud de permisividad en relación a determinados comportamientos y actitudes.

Es en este momento cuando se justifica la intervención de lo que designamos, como lo hacen otros autores, de Preventólogos, que cumplen los objetivos principales de prevención: intervenir para - resolver - solucionar vencer - evitar.

Y ¿cuál es el papel que le está realmente reservado a la familia? La familia amplia ya no predomina y la familia nuclear se ha instalado, reducida a un espacio relacional cada vez más pequeño. Hay un aumento de las familias monoparentales, sustitutas, urbanas, socialmente excluidas y familias ausentes (Patrício, L. 1998). "La precariedad de la relación, los cambios, han traído dificultades de comunicación, —más negativas que positivas-. una ruptura en los primeros contactos a favor de los contactos secundarios, con el respectivo debilitamiento de las relaciones de consaguineidad (parentesco), un declinar en la importancia social de la familia, de la desaparición del sentido de vecindad y la erosión de las bases tradicionales del bienestar social" (Patrício, L. 1997).

Es verdad que las escuelas y otras instituciones van asumiendo progresivamente el papel de la educación y ocupación de los niños y jóvenes. La organización y promoción de actividades recreativas, de juegos y actividades sociales, dependen cada vez menos de la intervención directa de la familia, por razones que todos reconocemos y que están suficientemente descritas en la literatura sociológica, y cada vez más de estructuras sociales organizadas. Esta pérdida de control puede ser vista como una descalificación de los padres, o puede originar una laguna educacional — "Síndrome de la pérdida de autoridad" (Antoni, M., Frase y Scotto, J.C.)

Sin embargo padres y educadores tienen y seguirán teniendo una importancia fundamental en la transmisión de valores y como modelos de identificación, en el desarrollo. La mejor opción, será, de este modo, ayudar a padres y educadores a través de una prevención familiar en este desafío de la educación.

Surgen entonces dos cuestiones: ¿Cómo pueden los padres ser coparticipantes activos y agentes preventivos? ¿Dónde pueden encontrar estos el apoyo y la experiencia para responder a sus propias dudas, miedos y 
ansiedades personales? Hay así una urgente necesidad de delinear e adoptar programas de prevención que respondan a la necesidad de reforzar a las familias con respuestas e instrumentos, haciendo de ellas también copartícipes en la prevención.

Recientemente, y por lo que se refiere a la intervención, la tendencia es desarrollar programas que aumenten y refuercen los modelos educativos de padres y educadores, añadiendo información, reduciendo el impacto de factores de riesgo y comprendiendo el potencial de los factores de protección. La importancia y la eficacia de estos programas de intervención precoz en la familia ya ha sido demostrada por varios autores (Kumpfer, 1996; Webster-Stratton, 1981, 1982, 1984; Szpocznik's, 1996; Farrington y Hawkins, 1991).

Es importante saber si los programas de que estamos hablando se adaptan a modelos educacionales y promotores de la salud, si son programas de prevención primaria específicos y si están disponibles.

Buena parte de la investigación en este campo deriva del estudio de programas canadienses y americanos. Así, de los cerca de 500 programas de Prevención Familiar ya evaluados por Kumpfer (1995), destacamos algunas conclusiones que se relacionan con los aspectos a considerar, para mejorar la eficacia en la delineación y conveniencia de los nuevos programas. Así, estos programas deberán ser:

a) Programas integrales:

El término integral encierra una definición clara y exacta del objetivo que se quiere alcanzar y de los resultados que se pretenden obtener, e implica usar los instrumentos correctos que permitan alcanzar estos objetivos. Es importante que las personas que reciben los mensajes puedan comprenderlos, para poderlos asimilar convenientemente y con eficacia.

b) Programas dirigidos a toda la familia e integrados en los diferentes momentos del ciclo familiar.

La eficacia de estos programas resulta de hacer partícipes a todos los miembros de la familia en las diversas actividades. Esta participación puede tener lugar en diferentes momentos para padres e hijos, pero la intervención de todos es vital, para que se sientan parte de un todo en la resolución de un problema y en la respuesta a las cuestiones, desafíos y objetivos a alcanzar.

c) Programas de larga duración

Por lo que se refiere a la prevención, el elemento tiempo es fundamental para activar la intervención de modo que modifique no sólo las actitudes sino también el comportamiento. Estos cambios sólo pueden tener éxito si tenemos tiempo para producirlos.

d) Basados en el conocimiento de los factores de riesgo y protección

En las acciones de prevención no basta intentar resolver las consecuencias físicas y psicológicas del consumo de sustancias. Es importante comprender y discutir todos los factores, actuales y/o remotos, relacionados con el uso de drogas (el funcionamiento de la familia, el papel de la escuela, éxito y fracaso escolar, la comunidad ...).

e) Adaptados a las necesidades de cada comunidad

Aunque los programas puedan ser delineados de una forma amplia, es esencial que, para que puedan ser usados universalmente, sean tenidas en cuenta las especificidades de cada comunidad/región y que los programas puedan ser adaptados a estas micro-realidades. Esto implica un rigurosos estudio a nivel social, cultural, económico, recreativo y ocupacional y el conocimiento del campo de la acción planeada (Mendes F. y al., 1998).

Basados en estos presupuestos y a partir de la estructura de la familia y de las necesidades de cada uno de sus miembros, Kumpfer (1995) definió cinco tipos diferentes de prevención familiar: 1)Escuela de padres; 2) Programas para el desarrollo de modelos familiares y educativos 3) Terapia familiar 4) Programas de intervención en situaciones de crisis familiar 5) Programas de rehabilitación intensiva para jóvenes com problemas con la Justicia.

\section{FACTORES DE RIESGOY DE PROTECCIÓN FAMILIAR}

Hoy sabemos cómo diferentes comportamientos y actitudes familiares pueden estar directamente relacionados con el uso de drogas por algunos de sus miembros, especialmente por los jóvenes.

Merikangas et al. (1998) hablan de la existencia de dos tipos de factores familiares que, de una manera u otra, pueden potenciar y, en algunos casos, desencadenar el consumo de sustancias en los jóvenes: los factores 
específicos y los factores no específicos. Los factores específicos son los que tienen una influencia directa en el uso/abuso de sustancias por los hijos: - exposición a las drogas en una fase prenatal del desarrollo - consumo de drogas por los padres - aceptación por parte de los padres del uso de drogas. La especificidad de estos factores la refuerzan autores como Duncan y al., (1995); Petterson, (1986); Barnes y Welte, (1986); Brook y al., (1986); Molina y al., (1994).

Son considerados factores no específicos: Desestructuración familiar - exposición a conflictos familiares (padres separados) - exposición a altos niveles de stress - psicopatologías familiares - negligencia - abuso físico, emocional y sexual - exclusión social.

La calidad relacional de una pareja no parece tener una influencia directa sobre el uso de drogas, pero cuando se combina con otros factores de riesgo, la probabilidad del uso de drogas por los jóvenes aumenta significativamente (Kaplan, 1995). El uso de sustancias por parte de los padres puede contribuir a una disfunción familiar que puede mantener el consumo o favorecer su incremento (Gabel y Schindlecker, 1991; McCarthy y Auglin, 1990).

Además, y refiriéndose a la relación parental, si Robins (1985) cita el hecho de que los conflictos familiares estén asociados a la delincuencia juvenil y al uso de drogas, Farringtion y al. (1985), van más lejos y consideran los conflictos familiares como el mayor riesgo en la salud de los adolescentes, relacionándolos con el uso de drogas.

Tanto las actitudes demasiado permisivas como las demasiado autoritarias por parte de los padres pueden estar directamente conectadas con el uso de drogas por parte de los hijos (Baumrind, 1993; Shelder y Block, 1990); también las actitudes permisivas aumentan el riesgo de alcoholismo o consumo de diversas sustancias por los hijos (Johnson y al., 1984; Barnes y Welte, 1986; Brook y al., 1986).

Cuanto mayor sea el número de miembros de la familia que use drogas o que implique a los niños en ese uso (incluso aunque esa implicación se reduzca, por ejemplo, a pedir al niño que vaya a comprar una cerveza o un paquete de tabaco), mayor será el riesgo de que los niños comiencen a usar drogas (Ahmed y al., 1984; Robins y Przybeck, 1985).

La falta de participación maternal en las actividades de los niños, (Kandel, 1990; Andrews, 1987), los efectos en la familia de consumos maternos, (Kandel, 1990), la indisciplina familiar (Plenning y Barnes, 1982; Baumrind, 1983), y la falta o inadecuación de aspiraciones en la formación de los niños, son también fuertes factores decisivos del uso. Brook y al. (1990) llegaron a la conclusión de que las técnicas de control materno son más importantes que las paternas en la explicación del uso de marihuana por parte de los jóvenes. Baumrind (1983) menciona las actitudes de falta de franqueza o gran permisividad como contribuciones para un elevado nivel de uso de drogas. Por otro lado Reilly (1979) en el estudio efectuado con familias de adolescentes que usaban drogas, evidencia factores de comunicación negativos (crítica y culpa), límites comportamentales inconsistentes y poco claros y también expectativas poco realistas de los padres en relación a los hijos.

También el papel del padre puede ser predictivo o preventivo del consumo de sustancias, dependiendo tanto de la manera de relacionarse afectivamente con sus hijos (Maccoby, 1984), como por su estilo de actuar y del modelo que desempeña: autoritario - permisivo - o indiferente. (Baumrid, 1980; Craig, 1996; Maccoby y Martin, 1983).

Algunos estudios han demostrado que los consumidores ven a sus padres más rechazadores y más hostiles que los no consumidores. Nolem-Hebeisen y al. (1984) han descubierto que la calidad de la relación entre padres e hijos influía sobre los modelos del uso; también el nivel de conflicto parece estar relacionado con el consumo, siendo un factor de riesgo importante (Rulter y Jiller, 1983; Farrington y al., 1985; Simcha-Fagan, Gerster y Langner, 1986).

Pero la relación entre padres e hijos también funciona como un factor de protección (Jorsuch y Butler, 1976; Jessor y Jessor, 1977; Kim, 1979; Nolem-Hebeisen y al., 1984; Brook y al., 1986; Selmow, 1987); implicación y dedicación son sinónimos de protección y freno de los jóvenes para el consumo de drogas. Hawkins y al., (1992), revelan que los fuertes lazos parentales pueden inhibir el uso de drogas y comportamientos delincuentes en los adolescentes.

Hundleby y Mercer (1987) aluden a la confianza, seguridad e implicación que sienten los niños como un factor de protección del consumo. Hirschi (1969) afirma que la ligazón a la familia puede inhibir el uso de drogas en la adolescencia. Otros autores como Patterson y al.,(1982) han llegado a la conclusión de que una decuada prevención paterna actuaba también como factor importante en la reducción de la delincuencia. Para Brook y al. (1990), " la interiorización de los valores tradicionales lleva al desarrollo de fuerte ligazón padres-hijos", lo que, a su vez, está asociado a modelos de no-uso de drogas". 


\section{CONCLUSIÓN}

Para terminar esta ya larga reflexión diremos que la delineación de futuros Programas de prevención familiar, deberá basarse en tres premisas fundamentales:

1. Conocimiento de las diferentes realidades familiares y su relación o correlación con el consumo de sustancias.

2. Perspectiva y expectativa de una mayor investigación sobre la familia, con identificación de los factores de riesgo y de los factores de protección.

3. Investigación y evaluación de todos los programas y acciones existentes que implican a las familias.

En cuanto al primer punto y teniendo presente todas las publicaciones e investigaciones, queremos hacer referencia a un estudio del IREFREA - 1998, en 4 países europeos (Portugal, España, Francia e Italia), que apunta hacia un refuerzo del establecimiento de normas y límites parentales, para evitar actitudes permisivas en relación a la disciplina y a comportamientos antisociales, realzando la importancia de los lazos afectivos en la familia Sabiendo que la importancia de la familia va disminuyendo a medida que los niños crecen, es fundamental reforzar tanto el papel de los padres al lado de los hijos más pequeños, como su comportamiento cuando estos son adolescentes.

En los diferentes momentos de los ciclos familiares debe la prevención intensificar estrategias que refuercen los diferentes factores de protección.

Así, durante la $1^{\mathrm{a}}$ y $2^{\mathrm{a}}$ infancia, ayudar en la transmisión de valores (sociales, familiares y religiosos), reforzando el papel parental, creando la conciencia de la importancia de cómo el comportamiento de los padres se refleja en las actitudes de los hijos, en el desarrollo de estrategias que les permitan a los padres expresar su capacidad afectiva con sus hijos, en la creación de espacios para establecer diálogos futuros a través de una comunicación directa, sincera y auténtica.

En la adolescencia, facilitar actividades, acciones y programas fundamentados en el conocimiento de los factores de riesgo y comportamientos de riesgo, que permitan potenciar los factores de protección, para evitar, reducir o retrasar los consumos. Ayudar a los adolescentes a crecer con autonomía e independencia, dentro de las normas y límites establecidos por sus progenitores.

Del segundo punto queremos subrayar la necesidad de poder disponer de datos cualitativos y cuantitativos sobre las familias, su funcionamiento o disfuncionamiento, de manera que se pueda cotejar y actualizar el conocimiento de los factores de riesgo y de los factores de protección. Discutir los modelos teóricos en que se basan las diversas intervenciones preventivas, de acuerdo con los objetivos que pretendemos alcanzar, sin olvidar nunca las realidades nacionales, regionales, sociales o culturales del lugar en que vamos a intervenir. Mayor profundización en el concepto de prevención y, consecuentemente, en los modelos a utilizar.

En relación al último punto, proponemos la creación de un Vademecum con las diferentes prácticas preventivas en Europa sobre la familia, a fin de que puedan ser conocidas, discutidas, comparadas y evaluadas. En este momento el Instituto Europeo de investigación sobre prevención IREFREA está llevando a cabo un proyecto de investigación que tiene lugar en cinco países europeos, para conocer y evaluar los programas de Prevención familiar. Este proyecto será el desencadenante de lo que pretendemos que llegue a ser una política global y concertada de Prevención familiar en la Comunidad Europea.

\section{BIBLIOGRAFÍA}

Ahmed S.W.; Bush P.; Davidson, F.R. and lannotti R.J. (1984). Predicting children's use and Intentions to use Abusable substances. Paper presented at the Annual meeting of the American Public Health Association, Anaheim, CA, November.

Barnes G.; and Welte J.(1986). Patterns and predictors of alcohol use among 1-12th grade students in New York State. Stud Alcohol 47: 53-62.

Baumrind D.(1983). Specious causal attributions in the social sciences: The reformulated stepping-stone. Theory of heroin use as examplar. Journal of Personality and social Psychology, 45: 1289-1289.

Baumrind D. (1980). New directons in socialization research. American Psychologist, 35: 639-650. 
Baumrind D. and Moselle K.A.(1985). A developmental perspective on adolescent drug abuse. Adv. Alcohol. Subst. Abuse 4: 41-67.

Bergeret J. (1982). Toxicomanie et Personalité. Presses Universitaires de France. Paris.

Brook J.S., Whiteman M., Nomura C.,Gordon A.S. and Cohen P.(1988). Personality, family and ecological influences on adolescent drug use: A developmental analysis. Adv. Alcohol Subst. Abuse. 5: 123-161.

Brook J.S., Gordon A.S., Whiteman M. and Cohen P.(1986). Some models and mechanisms for explaining the impact of maternal and adolescent characteristics on adolescent stage of drug use. Developmental Psychology. 22: 460-477..

Brook J.S., Brook D.W., Gordon A.S., Whiteman M. and Cohen, P.(1990). The psychosocial etiology of adolescence drug use: A family interactional approach. Genetic, Social and general Psychology Monographs. 116: 111-267.

Calafat A. (1995). Los factores de riesgo como fundamento de programas preventivos. E. Becoña, A. Rodrigues e I. Salazar. Drogodependencias. 4. Prevención: 75-103. Santiago de Compostela: Servicio de Publicaciones e Intercambio Científico de la Universidad de Santiago de Compostela.

Calafat A. (1998). Hacia una prevención institucionalizada y cientifica. Adicciones, Vol.10, n 4: 291-297.

Coie et al. (1993). The Science of Prevention. A Conceptual Framework and Some Directions for a National Research Program. American Psychologist. Oct. 1993: 1013-1022.

Duncan T.E., Duncan S.C., Hops,H. and Stoolmiller M.(1995). An analysis of the relationship between parent and adolescent marijuana use via generalised estimating equation methodology. Multivariate Behav Pres 30 (3): $317-339$.

Farrington D.P., Gallagher B.,Morley L., Ledger R.J., and West D.J. (1985). Cambridge Study in Delinquent Development: Longterm follow-up. First Annual Report to the Home Ofice, August, 13 Cambridge: Cambridge University, Institute of Criminology.

Gabel S. and SchindledeckerR.(1991). Agressive behavior in youth: Characteristics, outcome and psychiatric diagnosis. Journal American Academie Child Adolescence Psychiatry. 30(6):982-988.

Gordon R. (1987). An operational classification of disease prevention. In: Steinberg J.A., and Silverman M.M.. Eds. Preventing Mental Disorders. Rockville, MD: U.S Department of Health and Human Services,

Graig G.J.(1996). Desarrollo psicologico 7 ed. Mexico: Prince- Hall Hispanoamericana.

Hawkins J.D., Catalano R.F. and Miller J.Y.(1992). Risk and protetive factors for alcohol and other drug problems in adolescence and early adulthood: Implications for substance abuse prevention. Psychol. Bull. 112(1): 64-105.

Hirschi T.(1969). Causes of delinquency. Berkeley, CA: University of California Press.

Hundleby J.D. and Mercer G.W. (1987). Family and Friends as Social Environments and their Relationship to Young Adolescents'Use of Alcohol, Tobacco and Marijuana. Journal of Clinical Psychology. 44: 125-134.

Jessor R. et Jessor, S.L. (1977). Problem behaviour and psychosocial development: A longitudinal study of youth. New York: Academic Press.

Jessor R., Donovan J.E. and Windmer K.(1980). Psychosocial Factors in Adolescent Alcohol and Drug Use: The 1980 National Sample Study and the 1974-78 Penal Study. Unpublished final report. Boulder: University of Colorado, Institute of Behavioral Science.

Kaplan H.B.(1995). Drugs, Crime and other Deviant Adaptations. Longitudinal Studies. New York: Plenum.

Kim S. (1979). An Evaluation of Ombudsman Primary Prevention Program on Student Drug Abuse. Charlotte, N.C.:Charlotte Drug Education Center.

Kumpfer K.L. (1995). Access to hard-to-reach women: Interventions as confounds or startegy. In: Jones, C. and De la Rosa, M.; eds. National Institute on Drug Abuse Tachnical Review: Methodological Issues: Etiology and Consequences of Drug Abuse Among Women. Silver Spring, M.D. N.I.D.A..

Kumpfer K.L. (1996). Selective Preventive Interventions: The Strengthening Families Program".Paper presented at the National Insitute on Drug Abuse Technical Review onDrug Abuse Prevention Through Family Interventions, Gaithersburg, MD. January 25-26.

Maccoby E.E. (1984). Socialization and developmental change. Child Development,55, 317 - 328

Maccoby E.E. e Martin J.A. (1983). Socializations in the contest of the family: Parent-child interactions. Em P.H. Mussen ( Ed.) Handbook of child psychology. Vol.4. Socialization, personality and social development. New York: Wiley.

Maccoby E.E. ( 1992 ). The role of parents in the socialization of children: A historic overview. Developmental Psychology. 28: 1006-1017.

Martín E.. La prevención en Espagña hoy: Propuesta de consenso institucional, técnico y social. En Plan National sobre Drogas (Ed.) Prevención de las drogodependencias. Análisis y propuestas de actuación (pp. 67-84).Madrid: Delegación del Gobierno para el Plan Nacional sobre Drogas, Ministerio del Interior.

McCarthy W.J. and Anglin M.D.(1990). Narcotics addicts: Effet of family and parental risk factors on timing of emancipation drug use onset, pre-addiction incarcerations and educational achievement. Journal of Drug Issues. 20 (1): 99-123.

Mendes F., Relvas A.P.. Lourenço M., Reccio j. I. , Pietralunga S. Broyer G. Bussac M.H., Calafat A., Stocco P. (1999). Family Relationships and Primary Prevention of Drug Use in Early Adolescence. Eds IREFREA.

MeriKangas K.R., Decker L., Fenton B. (1998). Family Factors and substance abuse: implications for prevention. NIDA Research Monograph.177: 12-41. 
Molgaard V. and Kumpfer K.L.(1995). The lowa Strenghtening Families Program for Families with Pre and Early Teens. lowa State University, Ames IA.

Molina B.S.G., Chassin L., and Curran P.J.(1994). A comparaison of mechanism underlying substance use for early adolescent children of alcoholics and controls. Stud Alcohol. 55: 269-275.

Patricio L.D.B. (1997). Face à Droga como (Re)Agir. S.P.T.T. (Eds.). Lisboa.

Patricio L.D.B. (1998). Familia e Toxicodependência. Paper presented at IREFREA seminar in Coimbra.

Penning M. and Barnes G.E.(1982). Adolescent Marijuana Use: A review. International Journal of Addictions 17: $749-91$.

Patterson G.R.(1986). Performance models for antisocial boys. Am Psychol.. 41: 432-444.

Reilly D.M. (1979). Family Factors in the Etiology and Treatment of Youthfull Drug Abuse. Family Therapy.11: $149-71$.

Robins L.N. and Przybeck T.R.(1985). Age on onset of drug use as a factor in drug use and other disorders, in: Jones, C.L. and Battjes, R.J., eds. Etiology of Drug Abuse: Implications for Prevention. National Institute on Drug Abuse Research Monograph 56. DHHS,Pub.No.(ADM) 85-1335. Washington,DC: Sup. Of Docs., U.S. Govt. Print-Off., 1985, pp 178-193.

Roosa M.W., Beals J., Sandler I.N. and Pillow D.R.(1990). The role of risk and protective factors in predicting symptomotology in adolescent self-identified children of alcoholic parents. American Journal Community Psychologie.18: 725-741.

Rutter M. and Giller (1983). Juvenil Delinquency: Trends and Perspectives. New York: Penguin.

Selnow, G.W. (1987). Parent-Child Relationships in Single and two Parent Families: Implications for substance usage. Journal of Drug Education. 17: 315-26.

Shedler J. and Block J.(1990). Adolescent Drug use and psychological health: A longitudinal inquiry. Am. Psychol..45(5): 612630.

Simcha-Fagan O.,Gersten J.C. and Langner T.S. (1986). Early Precursors and Concurrent Correlators of Patterns of Ilicit Drug use. In Adolescence Journal of Drug Issues. 16: 7-28.

Szapocznik J. (1996). "Scientific findings that have emerged from family intervention research at the Spanish Family Guidance Center and the Center for Family Studies." Paper presented at the N.I.D.A. Technical Review on Drug Abuse Prevention Through Family Interventions, Gaithersburg, MD, January 25-26

Webster-Stratton C. (1981). Modification of mother's behaviors and attitudes trough video tape modeling group discussion. Behav. Ther. 12: 634-642.

Webster-Stratton C. (1981). Videotaping modeling: A method of parenting education. J. Clin. Child Psychol.. 10: 93-97.

Webster-Stratton C. (1982). Long-term effects of a video-tape modeling parent education program: Comparison of immediate and 1-year follow-up results. Behav. Ther. 13: 712-714.

Webster-Stratton C. (1982). Teaching mothers though videotape moeling to change their children's behaviors. J. Pediatr. Psychol.. 7: 279-294. 